Tropical Journal of Pharmaceutical Research April 2019; 18 (4): 897-905

ISSN: $1596-5996$ (print); 1596-9827 (electronic)

(C) Pharmacotherapy Group, Faculty of Pharmacy, University of Benin, Benin City, 300001 Nigeria.

\title{
Tryptophan-kynurenine pathway as a novel link between gut microbiota and schizophrenia: A review
}

\author{
Yaping Wang ${ }^{1-3}$, Xiuxia Yuan ${ }^{1-3}$, Yulin Kang ${ }^{4}$, Xueqin Song ${ }^{1-3 *}$ \\ ${ }^{1}$ The First Affiliated Hospital/Zhengzhou University, ${ }^{2}$ Biological Psychiatry International Joint Laboratory of Henan/Zhengzhou \\ University, ${ }^{3}$ Henan Psychiatric Transformation Research Key Laboratory/Zhengzhou University, Zhengzhou, ${ }^{4}$ Department of \\ Biomedical Engineering, College of Engineering, Peking University, Beijing, China
}

*For correspondence: Email: it71265@163.com

Sent for review: 27 December 2018

Revised accepted: 21 March 2019

\begin{abstract}
Gut microbiota and its metabolite tryptophan play an important role in regulating neurotransmission, immune homeostasis and oxidative stress which are critical for brain development. The kynurenine pathway is the main route of tryptophan catabolism. Kynurenine metabolites regulate many biological processes including host-microbiome communication, immunity and oxidative stress, as well as neuronal excitability. The accumulation of metabolites produced by kynurenine pathway in brain results in the activation of the immune system (increase in the levels of inflammatory factors) and oxidative stress (production of reactive oxygen species, ROS), which are associated with mental disorders, for example schizophrenia. Thus, it was hypothesized that perturbations in kynurenine pathway could cause activation of immunity, and that oxidative stress may be involved in the etiology of schizophrenia. The present work is a review of the latest studies on the possible role of kynurenine pathway in schizophrenia, and mechanism(s) involved.
\end{abstract}

Keywords: Gut microbiota, Kynurenine pathway, Inflammation, Oxidative stress

This is an Open Access article that uses a fund-ing model which does not charge readers or their institutions for access and distributed under the terms of the Creative Commons Attribution License (http://creativecommons.org/licenses/by/4.0) and the Budapest Open Access Initiative (http://www.budapestopenaccessinitiative.org/read), which permit unrestricted use, distribution, and reproduction in any medium, provided the original work is properly credited.

Tropical Journal of Pharmaceutical Research is indexed by Science Citation Index (SciSearch), Scopus, International Pharmaceutical Abstract, Chemical Abstracts, Embase, Index Copernicus, EBSCO, African Index Medicus, JournalSeek, Journal Citation Reports/Science Edition, Directory of Open Access Journals (DOAJ), African Journal Online, Bioline International, Open-J-Gate and Pharmacy Abstracts

\section{INTRODUCTION}

Schizophrenia is a severe neurodegenerative disease that affects about $1 \%$ of the population. The definite underlying mechanism of this disease is far from known. Accumulating data from both epidemiological investigation and clinical studies support the neurodevelopmental hypothesis of schizophrenia, and have identified specific dysfunctions in neurodevelopment that might be critical in the pathogenesis of the disease [1]. Gut microorganisms refer to a complex microbial ecosystem that has been identified as a key modulator of neurodevelopment which is very likely to be involved in the occurrence of schizophrenia [2].

The possible roles that gut microbiota play in brain development are as follows: In the first place, tryptophan is used in the production of numerous biologically important metabolites with significant physiological functions, and has critical implications for the development of the central nervous system (CNS). Serotonin (5hydroxytryptamine, HT) which is derived from tryptophan through the action of 
tryptophan hydroxylase (TrpH), modulates critical neurodevelopmental processes. These processes are regulated by short-chain fatty acids (SCFAs) secreted by gut microflora, and a role of SCFAs in the stimulation of the expression of the rate-limiting enzyme TrpH1 has been suggested [3]. Secondly, the neurotrophins (e.g. brain-derived neurotropic factor, BDNF) which are closely related to neurodevelopment and neuroprotection, have critical functions in neuronal survival, synaptic plasticity and cognitive function.

Evidence suggests that the metabolites produced by gut microbiome impose modulatory effects on the expressions of BDNF and other proteins in brain, thereby affecting neurodevelopment and cognitive function [4]. Thirdly, gut microbiota might also affect $\mathrm{N}$-methyl-D-aspartate receptor (NMDAR) expression and contribute to brain development [5].

Studies have linked neurotoxicity to the etiology of schizophrenia. The kynurenine pathway is possibly the link between gut microbiota and brain neurons, since several neuroactive compounds produced during this process may show neurotoxicity and affect physiological activity and brain health. This review focused on the link between kynurenine pathway and the etiology of schizophrenia, and the mechanism(s) involved. The major aspects reviewed were (1) gut microbiota participation in brain development through regulation of metabolism of tryptophan and serotonin (5hydroxytryptamine, 5-HT) and SCFAs; (2) the gut microbiota-tryptophan-kynurenine pathway and its bioactive metabolites; (3) the kynurenine pathway and its metabolites connected with inflammation and oxidative stress; (4) the relationship between schizophrenia and the kynurenine pathway; and (5) the possible role of gut microbiota-tryptophan-kynurenine pathway in the pathogenesis of schizophrenia (Figure 1).

\section{Gut microbiota and neurodevelopment}

The gastrointestinal tract which houses trillions of microbial cells constitutes a large microbial ecosystem in humans. More than a billion years of co-evolution between mammalian and microbiota has led to inter-dependency. Large numbers of microbial species reside in the gastrointestinal tract and play a critical role in the development and homeostasis in the immune system [6]. Dysbiosis of intestinal microbes, including imbalance in their composition and function, is associated with many psychiatric diseases [7]. Many studies are devoted to exploring potential association between pathogenesis of mental disorders and gut microbiota in order to improve the understanding of neuropsychiatric diseases while uncovering novel therapeutic and preventive strategies.

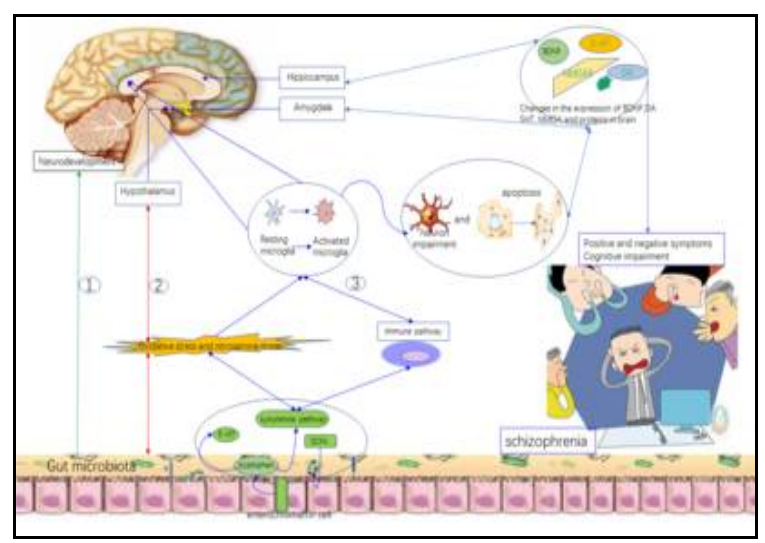

Figure 1: Gut microbiota is a key modulator of neurodevelopment. Gut microbiota regulates the secretion of tryptophan, 5-HT and production of SCFA which are important for brain health; Gut microbiota plays a significant role in the modulation of HPA axis which is involved in oxidative stress. Intestinal microbiota within the gut lumen regulates the synthesis and release of tryptophan by enterochromaffin cells (EC cells). Only a small portion of tryptophan is transformed to serotonin (5-HT)

The intestinal microbiome is a complicated ecosystem that varies between individuals, and is very likely to be a key modulator of neurodevelopment through the gut-brain axis. In studies on rodents, it was reported that alterations in the constitution and function of gut microbiota produce behavioral effects related to anxiety and depression, and cognitive impairment [8]. Germ-free mice display increased risk-taking behaviors, and show susceptibility to anti-anxiety and anti-depression [9]. These mice also exhibit learning and memory impairment, relative to conventional mice. Furthermore, GF mice displayed alterations in circulating total tryptophan levels [10], 5-hydroxytryptamine receptor $\left(5-\mathrm{HT}_{1 \mathrm{~A}}\right), \mathrm{BDNF}$, and NMDA receptors in the hypothalamus. These behavioral alterations were accompanied by changes in BDNF profiles in the hippocampus and amygdala, but these changes were reversed after colonization with microbiota of conventional mice [11].

Studies have shown that intestinal microorganisms regulate receptors for BDNF and NMDA which are neurotrophic factors and proteins essential for brain development and neural plasticity [5]. The response of the hypothalamuspituitary axis to the same external stress differs between specific pathogen-free (SPF) mice and germ-free mice [12]. It has been reported that 
Bifidobacterium preparation produced lower anxiety levels than placebo-fed MS mice, and also produced normal neurotransmitter levels in the hypothalamus [13]. These findings suggest that microbial colonization is essential for the development of the CNS which influences behavior. The possible reasons may be associated with metabolites such as tryptophan, $5-\mathrm{HT}$ and SCFA, as well as their secondary metabolites which are regulated by gut microbiota.

\section{Gut microbiota regulates tryptophan production}

Tryptophan is an essential amino acid, and so it must be provided in the diet. It is a substrate for the production of several substances with significant physiological functions. Tryptophanrich sources include eggs, fish, chocolate and meat. Gut microbiota exerts tremendous effects on the metabolism of tryptophan. They are capable of effecting direct uptake of tryptophan, thereby decreasing the amount of this essential amino acid available to their host. Mounting evidence indicate that the intestinal microbiota within the gut lumen influences the synthesis and release of serotonin regulated by enterochromaffin cells (EC cells) [14]. Serotonin, an important neurotransmitter derived from tryptophan degradation, participates in a variety of physical activities such as mood regulation, sleep, food consumption, pain and sex; and it has a critical role in CNS homeostasis [15].

Intestinal microbes are able to impact the amount of 5-HT synthesized and secreted by EC cells. Short chain fatty acids (SCFAs), just like acetate and butyrate produced by intestinal microbes, increase the mRNA expression of tryptophan hydroxylase $1(\mathrm{TpH} 1)$, the key enzyme involved in 5-HT synthesis [3]. The $\mathrm{TpH} 1$ expression and mucosal 5-HT levels are increased in GF mice after colonization with human intestinal microflora, indicating that gut microbiota may exert an effect on the function of EC cell via secretion of SCFAs [3]. In addition, 5-HT release from EC cells can be activated by stimulating mechanosensitive ion channels which play significant roles in this process. These findings indicate that the generation of SCFAs by gut microbiota may be an alternative approach to changing the availability of $5-\mathrm{HT}$, and in turn, secretion and motility in the gut.

\section{The gut microbiota-tryptophan-kynurenine pathway}

The amount of ingested tryptophan employed in anabolism is very small. In contrast, more than
95 percent of gut tryptophan is metabolized through the kynurenine pathway in mammals (Figure 2). This process produces several bioactive compounds (e.g. kynurenine, 3hydroxykynurenine, indoles, and $\mathrm{NAD}^{+}$) which participate in immunity and function as excitatory neurotransmitters. Since levels of enzymes of the kynurenine pathway tend to be much higher in peripheral tissues than in brain tissue, their roles in the kynurenine pathway exert local and systemic impacts.

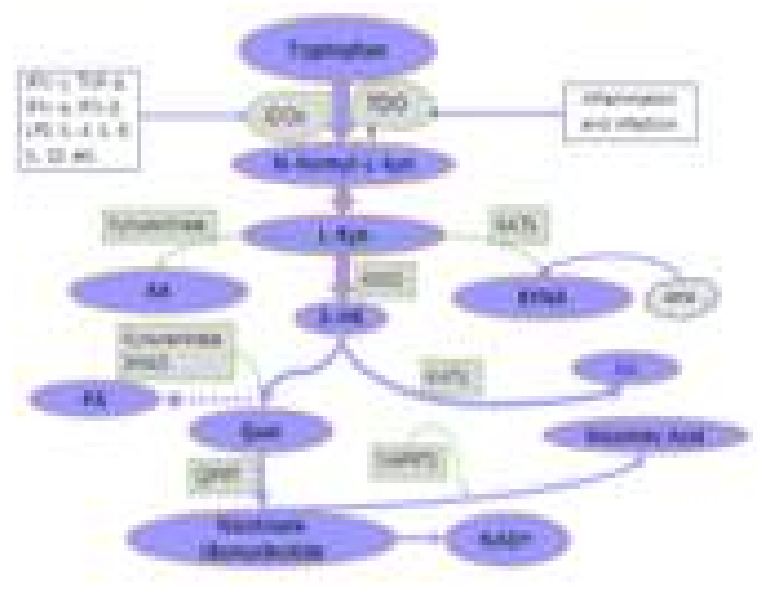

Figure 2: Kynurenine pathway. KMO: kynurenine monooxygenase; IDOs: indoleamine 2; 3dioxygenases; TDOs: tryptophan 2, 3-dioxygenase; NFormyl-L-kyn: N-formylkynurenine, L-Kyn: kynurenine; 3-HK: 3-hydroxykynurenine, KATs: kynurenineaminotransferases, XA: xanthranilic acid, PA: picolinic acid; Quin: quinolinic acid; NAPRT1: nicotinate phosphoribosyl transferase; $\mathrm{NAD}^{+}$: nicotinamide adenine dinucleotide

The catabolism of tryptophan in the brain, liver, kidney is quite complicated. This process generates many bioactive molecules that perform important physiological functions. The initial and rate-limiting step of kynurenine pathway, the transformation of tryptophan to $\mathrm{N}$ formylkynurenine, is under the catalysis of tryptophan 2, 3-dioxygenase (TDO2) and indoleamine 2, 3-dioxygenase (IDO-1 and IDO-2) [16]. N-Formylkynurenine is decomposed to kynurenine which undergoes hydroxylation, transamination and hydrolysis to yield anthranilate [17-19]. Kynurenine-3monooxygenase (KMO) has a very low Michaelis constant $\left(k_{m}\right)$ for kynurenine. Thus, under normal conditions, $\mathrm{KMO}$ is considered to be the primary metabolic route of the kynurenine pathway. This pathway results in the production of $\mathrm{NAD}^{+}$[20]. However, under specific conditions, kynurenine is transformed to kynurenic acid (KYNA) via the KAT route: KAT II may be important in the synthesis of most of the brain KYNA [19]. 
Kynurenine can also be metabolized to anthranilic acid by kynureninase and then further converted to 3-hydroxyanthranilic acid. However, the $k_{m}$ of kynureninase for kynurenine is so high that the kynureninase branch is activated only with tryptophan or kynurenine loading. This pathway contributes significantly to the endogenous generation of $\mathrm{NAD}^{+}$, and influences the neurotransmission of glutamatergic pathway as well as the redox reactions through its neuroactive metabolites.

Some intermediates of the pathway may also affect both the function and dysfunction of neurons and the brain, mainly through oxidative stress and inflammation [21]. However, their functions in the neuronal physiology and pathophysiology are still not clearly proposed. A sustained homeostasis in neuroactive metabolites of the KYN pathway is critical for the normal neural function. In contrast, several abnormalities in the levels of these metabolites have been presented in various tissues and brain regions in neuropsychiatric disorders, including schizophrenia.

\section{Metabolites of kynurenine pathway}

\section{Quinolinic acid}

Quinolinic acid (quin) produced by microglia is involved in neuronal excitotoxicity. Its neuronal excitatory properties are verified by using selective NMDA receptor antagonists. Tryptophan and the metabolites of the kynurenine pathway such as kynurenine and 3hydroxykynurenine (3-HK) are translocated from circulation to the brain across the BBB, and they contribute to the generation of microglia quin which generates detrimental effects linked to oxidative stress and apoptosis [22]. Quin also plays a role in stimulation of $\mathrm{Fe}^{2+}$-dependent lipid peroxidation, and the endogenous ions enhance the binding of quin to $\mathrm{N}$-methyl-D-aspartate receptor (MDAR) [23]. Evidence demonstrates that intra-cerebro-ventricular injection of quin in mice affects the function of CNS through induction of behavioral abnormalities [24].

\section{Kynurenic acid}

The KYNA produced by microglia in the kynurenine pathway is a neuroactive metabolite that antagonizes NMDAR [25]. Astrocytes also produce KYNA as the antagonist of NMDAR and alpha-7 nicotinic acetylcholine receptor ( $\alpha 7 n A C h R)$ [26]. This indicates the regulation of a7nAChRs, NMDAR, and their associated biological roles by the levels of endogenous KYNA. Studies have shown that KYNA regulates local inflammation mostly by GPR35 activation on the surface of immune cells in gastrointestinal tract [27]. In particular, the peripheral monocytes present the highest expression of GPR35. In addition, KYNA-GPR35 interaction is closely associated with immune homeostasis [27].

Alterations in levels of brain KYNA generate distinct behavioral effects, especially related to cognition. Studies have reported that high levels of KYNA in the brain might lead to cognitive impairment in rodents [28]. Further studies revealed that increases in the levels of KYNA in brain may contribute to presynaptic inhibition of $\alpha 7 n A C h R$ signaling, leading to abnormalities in behavioral responses and executive function [29]. On the other hand, decreases in the levels of KYNA in brain induced using KAT II knockout or agents that inhibit KAT II, led to improvements in related cognitive functions such as spatial memory, working memory and contextual learning, as well as behavior [30]. Several impairments in cognitive function are induced by elevation in forebrain KYNA levels following systemic administration of kynurenine [28].

In another study, regions of forebrain in rodents exhibited significant increases in KYNA, causing rapid reductions in extracellular dopamine and glutamine concentrations [31]. However, inhibition of KYNA synthesis increases the levels of these neurotransmitters, as well as the levels of acetylcholine. Besides, exposure to exogenous kynurenine, or elevations in brain KYNA caused pharmacologically, influences dopaminergic neuronal discharge in the CNS [32]. Therefore, it can be speculated that these behaviors and cognitive changes are associated with dopaminergic, cholinergic and glutamatergic neurotransmission.

\section{Kynurenine, 3-OH-anthranilic acid and AA}

In vivo studies have indicated that kynurenine is associated with immune stasis. Mounting evidence indicate that the activation of $A h R$ in cancer cells by kynurenine promotes cancer invasion and immune escape [33], and upregulates the expressions of genes that facilitate cell migration. The Kyn-AhR axis is thought to be a probable link between the activation of immune system and the proliferation of cancer cells. It has been reported that the metabolites of anthranilic acid activate metabotropic glutamate receptors [34]. Free radicals mediate the production of 3-HANA by $\mathrm{KMO}$, and 3-HANA is linked to oxidative stress and inflammation, leading to increased generation of superoxide anion [35]. 
Association of the kynurenine pathway with inflammation and oxidative stress

\section{Inflammation}

The kynurenine pathway modulates innate and adaptive immune responses. Studies found that kynurenine metabolites such as 3-HANA and quin induce apoptosis in thymocytes and Th1 helper cells, but not in Th2 helper cells [36]. Compounds derived from kynurenine pathway and tryptophan inhibited antigen-induced T-cell multiplication, and enhanced T-cell necrosis [37]. The rate-limiting enzymes of the kynurenine pathway (IDOs and GPR35) are highly expressed on the surface of dendritic cells responsible for immune tolerance. Thus, IDO might also play a key regulatory role with respect to T-cell-mediated immunity [38].

The kynurenine pathway is highly regulated by the immune system. The levels of enzymes are much higher in peripheral nervous system than in the central nervous system, and quin produced by the macrophages is 20 -fold more than that produced by microglial cells. About $60 \%$ of kynurenine in the peripheral tissue is transported into the brain. This is of great significance for microphage penetration across the BBB in the brain. As a key enzyme of kynurenine pathway, IDO is induced by several factors such as interferon- $\gamma$ (IFN- $\gamma$ ), tumor necrosis factor- $\alpha$ (TNF- $\alpha$ ) and infections [38]. This pathway becomes active in the presence of proinflammatory factors, but it is inhibited by antiinflammatory cytokines. In addition, animal and human studies have suggested that $\mathrm{KMO}$ is activated by inflammatory factors [39]. Consequently, this pathway is closely linked to the stability of the immune system.

\section{Oxidative stress}

It is generally believed that the main function of the kynurenine pathway is the production of $\mathrm{NAD}^{+}$, which is related to regulation of key oxidative stress events [40]. Higher expression of $\mathrm{KMO}$ leads to formation of intermediate products that activate glutamate receptors and lead to oxidative stress [41].

Kynurenine is metabolized by $\mathrm{KMO}$ to $3-\mathrm{HK}$ (Figure 1). In peripheral nervous system, 3-HK is translocated across the BBB, thereby increasing its bioavailability in the brain. In vivo and in vitro studies have found that 3-HK-mediated neuronal cell death is mitigated by the antioxidants glutathione, catalase and deferoxamine, demonstrating that the toxic effects of $3-\mathrm{HK}$ are exerted through imposition of oxidative stress
[42]. It would appear that 3-HK performs two opposite functions: a favorable function at the initial developmental period, and an unfavorable role during long-term exposure to harmful environments. The molecular mechanisms underlying these activities are not yet ascertained.

Quinolinic acid and kynurenic acid form an important pair that maintains balance in levels of oxidative stress [43]. The neurotoxicity of quinolinic acid occurs via increased glutamate signaling, lipid peroxidation and its effect as an NMDAR agonist [44]. Elevation of quinolinic acid leads to the activation of NMDAR. Results from animal studies have confirmed that quinolinic acid induces neuro-inflammation and oxidative stress in rats which may cause neurodegeneration in the striatum [45]. Kynurenic acid inhibits the neurotoxic effect of quinolinic acid and physically protects the neurons [46]. Therefore, homeostasis of the kynurenine pathway metabolites is necessary for maintenance of normal brain function.

\section{Schizophrenia and kynurenine pathway}

Schizophrenia is a severe mental disorder with high recurrence rate. The hyperactivity of dopamine system in brain plays a dominant role in the etiology of schizophrenia. However, the hypothesis of glutamatergic signaling in schizophrenia has gradually aroused great attention in recent years. Application of phencyclidine and ketamine induces clinical presentations consistent with schizophrenia in healthy subjects, and aggravates mental symptoms in schizophrenic individuals by antagonizing the glutamatergic N-methyl-Daspartate (NMDA) receptors [47]. The induction of schizophrenia with phencyclidine and ketamine created awareness that the kynurenine pathway is very likely to be associated with the pathogenesis of schizophrenia since one of its metabolites (KYNA) is an NMDAR antagonist.

Kynurenic acid (KYNA) which is produced in this pathway from a reaction catalyzed by kynurenine aminotransferases (KAT), antagonizes the NMDA receptor and $\alpha 7 n A C h R s$ in the CNS [48]. Mounting evidence have demonstrated increased KYNA levels in the cerebrospinal fluid and encephalic regions of schizophrenic subjects; these contribute to hypofunction of NMDA receptor, cognitive function impairment, and negative symptoms related to schizophrenia [49]. It has been shown that increased brain KYNA levels in rats are closely related to accentuated release of DA in midbrain and turbulence in prepulse inhibition, a deficit observed in 
schizophrenics, although reports from different studies vary [50]. For example, a few studies confirm that KYNA protects the neurons from oxidant damage by eliminating free radicals, thereby shielding them from excitotoxic injury [51]. It is suspected that KYNA neutralizes the neurotoxicity produced by quin, 3-HK and 3HANA. However, the mechanism underlying the role of KYNA in schizophrenia needs further confirmation.

Kynurenine lowers the activity of natural killer cells and dendritic cells, regulates the release of inflammatory cytokines, and promotes monocyte extravasation. This metabolite is associated with several neuropsychiatric disorders and neurodegenerative disease [52]. Upregulation of TDO expression increases kynurenine, and studies have indicated increases in kynurenineto-tryptophan ratio in the brain and cerebrospinal fluid of schizophrenia subjects [52]. Elevations in these markers are very likely due to consequences of peripheral inflammation and neuronal inflammation, which are involved in the pathogenesis of schizophrenia. Dysregulation in the metabolic pathway of tryptophan has been implicated in the pathogenesis of schizophrenia, based on the association between kynurenine pathway and inflammation.

The key enzyme of the tryptophan/kynurenine pathway (IDO) is induced in rodents by inflammatory factors. The function of IDO, apart from metabolizing tryptophan in the microenvironment to protect the host from infection, involves generating a variety of metabolites that restrain T-cell-mediated immune reaction against self-antigens [53]. A report indicated that exposure to IFN-y led to increase in the transcription of IDO in immune cells, dendritic cells, monocytes and macrophages [54]. The expression of IDO can also be regulated by IL-4, IL-6, IL-10 and TNF- $\alpha$ [55]. Moreover, infection-induced pro-inflammatory status of mothers during pregnancy increases the susceptibility of the offspring to schizophrenia during the adult period [56]. The inflammatory process is supposed to be associated with the etiology of schizophrenia in at least a subgroup of patients. Those inflammatory factors i.e. IL-6, TNF- $\alpha$ and IFN- $\gamma$ involved in the pathogenesis of schizophrenia participate in the activation of the kynurenine pathway [57] (Figure 2).

Evidence has demonstrated the possible involvement of the kynurenine pathway in schizophrenic presentations. Rats injected ketamine showed schizophrenic-like behaviors which were alleviated by the inhibitors of TDO, IDO and KMO. In addition, the inhibitors of these enzymes have been shown to block oxidative stress and lipid peroxidation, and increase the levels of antioxidants including superoxide dismutase (SOD) and catalase (CAT) in the prefrontal cortex (PFC), hippocampus and striatum [58]. Significant impairments of kynurenine route have been reported in rats with increased risk for neurodegenerative alterations and oxidative stress; this may be a possible mechanism linking schizophrenia to the kynurenine pathway [59]. Lipid peroxidation is enhanced by quinolinic acid at an early stage of tissue culture; it has been reported that elevations in quinolinic acid led to ROS-pathway necrosis in natural killer cells [60].

\section{CONCLUDING REMARKS}

The function of the kynurenine route of tryptophan metabolism in schizophrenia is constantly being explored, and a large number of studies have shown that endogenous kynurenine directly contributes to the etiology of neuropathology. The kynurenine pathway is the main route of tryptophan catabolism. This pathway is activated by inflammation and oxidative stress markers, and it exerts an impact on immunity and oxidative stress. In this context, understanding the role of intestinal microflora in controlling tryptophan availability and kynurenine metabolism is crucial for enhanced appreciation of the pathogenesis of schizophrenia.

It has been hypothesized that schizophrenia is associated with human gut dysbiosis with microbiota that secrete lipopolysaccharide endotoxins into the blood, resulting in damaged gut barrier integrity. Furthermore, it was suspected that small molecule toxins enter the peripheral blood, cause inflammatory reactions in the peripheral and central regions, and induce transcription of IDO in immune cells such as microglia, thereby impacting on kynurenine metabolism.

These processes may result in neural impairment and apoptosis, which then lead to changes in neurotransmission and abnormalities in communication between brain networks, ultimately resulting in schizophrenia. In all, there are still insufficient data on the mechanism by which intestinal flora and kynurenine pathway are linked to schizophrenia. Studies have shown that intestinal flora affects the metabolism of kynurenine through inflammation state and oxidative stress. However, the mechanism is far from clear, particularly where confounding factors are involved. 


\section{DECLARATIONS}

\section{Acknowledgement}

This study was supported by the National Natural Science Foundation of China (No.81571318 to XQS).

\section{Conflict of interest}

No conflict of interest is associated with this work.

\section{Contribution of authors}

We declare that this work was done by the author(s) named in this article and all liabilities pertaining to claims relating to the content of this article will be borne by the authors. All authors read and approved the manuscript for publication. Yaping Wang conceived and designed the study, Yaping Wang, Xiuxia Yuan, Yulin Kang, Xueqin Song collected and analysed the data, Xueqin Song wrote the manuscript. Yaping Wang and Xiuxia Yuan contributed equally to this work and should be considered as co-first authors.

\section{Open Access}

This is an Open Access article that uses a funding model which does not charge readers or their institutions for access and distributed under the terms of the Creative Commons Attribution License (http://creativecommons.org/licenses/by/ 4.0) and the Budapest Open Access Initiative (http://www.budapestopenaccessinitiative.org/rea d), which permit unrestricted use, distribution, and reproduction in any medium, provided the original work is properly credited.

\section{REFERENCES}

1. Birnbaum R, Weinberger DR. Genetic insights into the neurodevelopmental origins of schizophrenia. Nat Rev Neurosci 2017; 18(12): 727-740.

2. Kim S, Kim H, Yim YS, Ha S, Atarashi K, Tan TG, Longman RS, Honda K, Littman DR, Choi GB, et al. Maternal gut bacteria promote neurodevelopmental abnormalities in mouse offspring. Nature 2017; 549(7673): 528-532.

3. Reigstad CS, Salmonson CE, Rainey JF, Szurszewski $J H$, Linden DR, Sonnenburg JL, Farrugia G, Kashyap $P C$. Gut microbes promote colonic serotonin production through an effect of short-chain fatty acids on enterochromaffin cells. Faseb J 2015; 29(4): 1395-1403.

4. Frohlich EE, Farzi A, Mayerhofer R, Reichmann F, Jacan A, Wagner B, Zinser E, Bordag N, Magnes $C$, Frohlich
$E$, et al. Cognitive impairment by antibiotic-induced gut dysbiosis: Analysis of gut microbiota-brain communication. Brain Behav Immun 2016; 56: 140-155.

5. Maqsood R, Stone TW. The Gut-Brain Axis, BDNF, NMDA and CNS Disorders. Neurochem Res 2016; 41(11): 2819-2835.

6. Mao K, Baptista AP, Tamoutounour S, Zhuang L, Bouladoux N, Martins AJ, Huang Y, Gerner MY, Belkaid $Y$, Germain RN. Innate and adaptive lymphocytes sequentially shape the gut microbiota and lipid metabolism. Nature 2018; 554(7691): 255-259.

7. Grochowska M, Wojnar M, Radkowski M. The gut microbiota in neuropsychiatric disorders. Acta Neurobiol Exp (Wars) 2018; 78(2): 69-81.

8. Zheng $P$, Zeng B, Zhou C, Liu M, Fang Z, Xu X, Zeng L, Chen J, Fan S, Du X, et al. Gut microbiome remodeling induces depressive-like behaviors through a pathway mediated by the host's metabolism. Mol Psychiatry 2016; 21(6): 786-796.

9. Luo Y, Zeng B, Zeng L, Du X, Li B, Huo R, Liu L, Wang $H$, Dong $M$, Pan J, et al. Gut microbiota regulates mouse behaviors through glucocorticoid receptor pathway genes in the hippocampus. Transl Psychiatry 2018; 8(1): 187.

10. Mardinoglu A, Shoaie S, Bergentall M, Ghaffari $P$, Zhang $C$, Larsson E, Backhed $F$, Nielsen J. The gut microbiota modulates host amino acid and glutathione metabolism in mice. Mol Syst Biol 2015; 11(10): 834.

11. Bercik P, Denou E, Collins J, Jackson W, Lu J, Jury J, Deng $Y$, Blennerhassett $P$, Macri J, McCoy $K D$, et al. The intestinal microbiota affect central levels of brainderived neurotropic factor and behavior in mice. Gastroenterology 2011; 141(2): 599-609.

12. Huo R, Zeng B, Zeng L, Cheng K, Li B, Luo Y, Wang $H$, Zhou $C$, Fang $L$, Li $W$, et al. Microbiota Modulate Anxiety-Like Behavior and Endocrine Abnormalities in Hypothalamic-Pituitary-Adrenal Axis. Front Cell Infect Microbiol 2017; 7: 489 .

13. Moya-Perez A, Perez-Villalba A, Benitez-Paez A, Campillo I, Sanz Y. Bifidobacterium CECT 7765 modulates early stress-induced immune, neuroendocrine and behavioral alterations in mice. Brain Behav Immun 2017; 65: 43-56.

14. Le Beyec J, Pelletier A L, Arapis K, Hourseau M, Cluzeaud F, Descatoire V, Ducroc R, Aparicio T, Joly $F$, Couvelard $A$, et al. Overexpression of gastric leptin precedes adipocyte leptin during high-fat diet and is linked to 5HT-containing enterochromaffin cells. Int $\mathrm{J}$ Obes (Lond) 2014; 38(10): 1357-1364.

15. Moutkine I, Collins E L, Bechade C, Maroteaux $L$. Evolutionary considerations on 5-HT2 receptors. Pharmacol Res 2018; 22(3): 54-57.

16. Kanai M, Funakoshi H, Takahashi H, Hayakawa $T$, Mizuno S, Matsumoto K, Nakamura T. Tryptophan 2,3dioxygenase is a key modulator of physiological neurogenesis and anxiety-related behavior in mice. Mol Brain 2009; 2: 8.

Trop J Pharm Res, April 2019; 18(4): 903 
17. Allison DJ, Ditor DS. The common inflammatory etiology of depression and cognitive impairment: a therapeutic target. J Neuroinflammation 2014; 11: 151.

18. Lugo-Huitron R, Ugalde Muniz P, Pineda B, PedrazaChaverri J, Rios C, Perez-de la Cruz V. Quinolinic acid: an endogenous neurotoxin with multiple targets. Oxid Med Cell Longev 2013; 2013: 104024.

19. Schwarcz R, Bruno JP, Muchowski PJ, Wu HQ. Kynurenines in the mammalian brain: when physiology meets pathology. Nat Rev Neurosci 2012; 13(7): 465477.

20. Maddison D C, Giorgini $F$. The kynurenine pathway and neurodegenerative disease. Semin Cell Dev Biol 2015; 40: 134-141.

21. Wang $Q$, Liu $D$, Song $P$, Zou $M H$. Tryptophan-kynurenine pathway is dysregulated in inflammation, and immune activation. Front Biosci (Landmark Ed) 2015; 20: 11161143.

22. Sahm F, Oezen I, Opitz CA, Radlwimmer B, von Deimling A, Ahrendt T, Adams S, Bode HB, Guillemin GJ, Wick $W$, et al. The endogenous tryptophan metabolite and $N A D+$ precursor quinolinic acid confers resistance of gliomas to oxidative stress. Cancer Res 2013; 73(11): 3225-3234.

23. Parasram K. Phytochemical treatments target kynurenine pathway induced oxidative stress. Redox Rep 2018; 23(1): 25-28.

24. Ganzella M, Faraco RB, Almeida RF, Fernandes VF, Souza DO. Intracerebroventricular administration of inosine is anticonvulsant against quinolinic acid-induced seizures in mice: an effect independent of benzodiazepine and adenosine receptors. Pharmacol Biochem Behav 2011; 100(2): 271-274.

25. Perkins MN, Stone TW. An iontophoretic investigation of the actions of convulsant kynurenines and their interaction with the endogenous excitant quinolinic acid. Brain Res 1982; 247(1): 184-187.

26. Hilmas C, Pereira EF, Alkondon M, Rassoulpour A, Schwarcz R, Albuquerque EX. The brain metabolite kynurenic acid inhibits alpha7 nicotinic receptor activity and increases non-alpha7 nicotinic receptor expression: physiopathological implications. J Neurosci 2001; 21(19): 7463-7473.

27. Agudelo LZ, Ferreira DMS, Cervenka I, Bryzgalova G, Dadvar S, Jannig PR, Pettersson-Klein AT, Lakshmikanth T, Sustarsic EG, Porsmyr-Palmertz M, et al. Kynurenic Acid and Gpr35 Regulate Adipose Tissue Energy Homeostasis and Inflammation. Cell Metab 2018; 27(2): 378-392.

28. Pocivavsek A, Thomas MA, Elmer GI, Bruno JP, Schwarcz $R$. Continuous kynurenine administration during the prenatal period, but not during adolescence, causes learning and memory deficits in adult rats. Psychopharmacol (Berl) 2014; 231(14): 2799-2809.

29. Flores-Barrera $E$, Thomases DR, Cass DK, Bhandari A, Schwarcz R, Bruno JP, Tseng KY. Preferential Disruption of Prefrontal GABAergic Function by Nanomolar Concentrations of the alpha7nACh Negative
Modulator Kynurenic Acid. J Neurosci 2017; 37(33): 7921-7929.

30. Pocivavsek A, Wu HQ, Potter MC, Elmer Gl, Pellicciari R, Schwarcz R. Fluctuations in endogenous kynurenic acid control hippocampal glutamate and memory. Neuropsychopharmacol 2011; 36(11): 2357-2367.

31. Wu $H Q$, Okuyama M, Kajii $Y$, Pocivavsek A, Bruno JP, Schwarcz R. Targeting kynurenine aminotransferase II in psychiatric diseases: promising effects of an orally active enzyme inhibitor. Schizophr Bull 2014; 40(2): 152-158.

32. Linderholm KR, Alm MT, Larsson MK, Olsson SK, Goiny $M$, Hajos $M$, Erhardt $S$, Engberg $G$. Inhibition of kynurenine aminotransferase II reduces activity of midbrain dopamine neurons. Neuropharmacol 2016; 102: 42-47.

33. Cheong JE, Sun L. Targeting the IDO1/TDO2-KYN-AhR Pathway for Cancer Immunotherapy - Challenges and Opportunities. Trends Pharmacol Sci 2018; 39(3): 307325.

34. Curto $M$, Lionetto $L$, Negro $A$, Capi $M$, Fazio $F$, Giamberardino MA, Simmaco M, Nicoletti F, Martelletti $P$. Altered kynurenine pathway metabolites in serum of chronic migraine patients. J Headache Pain 2015; 17: 47.

35. Bohar Z, Toldi J, Fulop F, Vecsei L. Changing the face of kynurenines and neurotoxicity: therapeutic considerations. Int J Mol Sci 2015; 16(5): 9772-9793.

36. Fallarino $F$, Grohmann U, Vacca $C$, Bianchi R, Orabona C, Spreca A, Fioretti MC, Puccetti P. T cell apoptosis by tryptophan catabolism. Cell Death Differ 2002; 9(10): 1069-1077.

37. Byakwaga H, Boum Y, Huang Y, Muzoora C, Kembabazi A, Weiser SD, Bennett J, Cao H, Haberer JE, Deeks $S G$, et al. The kynurenine pathway of tryptophan catabolism, CD4+ T-cell recovery, and mortality among HIV-infected Ugandans initiating antiretroviral therapy. J Infect Dis 2014; 210(3): 383-391.

38. O'Connor JC, Andre C, Wang Y, Lawson MA, Szegedi SS, Lestage J, Castanon N, Kelley KW, Dantzer R. Interferon-gamma and tumor necrosis factor-alpha mediate the upregulation of indoleamine 2,3dioxygenase and the induction of depressive-like behavior in mice in response to bacillus CalmetteGuerin. J Neurosci 2009; 29(13): 4200-4209.

39. Connor TJ, Starr N, O'Sullivan JB, Harkin A. Induction of indolamine 2,3-dioxygenase and kynurenine 3monooxygenase in rat brain following a systemic inflammatory challenge: a role for IFN-gamma? Neurosci Lett 2008; 441(1): 29-34.

40. Massudi H, Grant R, Guillemin GJ, Braidy N. NAD+ metabolism and oxidative stress: the golden nucleotide on a crown of thorns. Redox Rep 2012; 17(1): 28-46.

41. Rios C, Santamaria A. Quinolinic acid is a potent lipid peroxidant in rat brain homogenates. Neurochem Res 1991; 16(10): 1139-1143.

42. Colin-Gonzalez AL, Maldonado PD, Santamaria A. 3Hydroxykynurenine: an intriguing molecule exerting dual 
actions in the central nervous system. Neurotoxicology 2013; 34: 189-204.

43. Garcia-Lara L, Perez-Severiano F, Gonzalez-Esquivel D, Elizondo G, Segovia J. Absence of aryl hydrocarbon receptors increases endogenous kynurenic acid levels and protects mouse brain against excitotoxic insult and oxidative stress. J Neurosci Res 2015; 93(9): 14231433.

44. Bryleva EY, Brundin L. Kynurenine pathway metabolites and suicidality. Neuropharmacology 2017; 112(2): 324330.

45. Mishra J, Kumar A. Improvement of mitochondrial function by paliperidone attenuates quinolinic acidinduced behavioural and neurochemical alterations in rats: implications in Huntington's disease. Neurotox Res 2014; 26(4): 363-381.

46. Cervenka I, Agudelo LZ, Ruas JL. Kynurenines: Tryptophan's metabolites in exercise, inflammation, and mental health. Sci 2017; 357(6349): 9794.

47. Yasuda K, Hayashi $Y$, Yoshida $T$, Kashiwagi $M$, Nakagawa N, Michikawa T, Tanaka M, Ando R, Huang $A$, Hosoya $T$, et al. Schizophrenia-like phenotypes in mice with NMDA receptor ablation in intralaminar thalamic nucleus cells and gene therapy-based reversal in adults. Trans/ Psychiatry 2017; 7(2): 1047.

48. Obara-Michlewska M, Tuszynska P, Albrecht J. Ammonia upregulates kynurenine aminotransferase II mRNA expression in rat brain: a role for astrocytic NMDA receptors? Metab Brain Dis 2013; 28(2): 161-165.

49. Linderholm KR, Skogh E, Olsson SK, Dahl ML, Holtze M, Engberg G, Samuelsson M, Erhardt S. Increased levels of kynurenine and kynurenic acid in the CSF of patients with schizophrenia. Schizophr Bull 2012; 38(3): 426432.

50. Condray R, Dougherty GG, Jr., Keshavan MS, Reddy $R D$, Haas GL, Montrose DM, Matson WR, McEvoy J, Kaddurah-Daouk R, Yao JK. 3-Hydroxykynurenine and clinical symptoms in first-episode neuroleptic-naive patients with schizophrenia. Int $J$ Neuropsychopharmacol 2011; 14(6): 756-767.

51. Mackay GM, Forrest CM, Stoy N, Christofides J, Egerton M, Stone TW, Darlington L G. Tryptophan metabolism and oxidative stress in patients with chronic brain injury. Eur J Neurol 2006; 13(1): 30-42.

52. Kegel ME, Bhat M, Skogh E, Samuelsson M, Lundberg K, Dahl ML, Sellgren C, Schwieler L, Engberg G, Schuppe-Koistinen $I$, et al. Imbalanced kynurenine pathway in schizophrenia. Int J Tryptophan Res 2014; 7: 15-22.

53. Munir S, Larsen SK, Iversen TZ, Donia M, Klausen TW, Svane IM, Straten PT, Andersen MH. Natural CD4+ Tcell responses against indoleamine 2,3-dioxygenase. PLoS One 2012; 7(4): 34568.

54. Taylor MW, Feng GS. Relationship between interferongamma, indoleamine 2,3-dioxygenase, and tryptophan catabolism. Faseb J 1991; 5(11): 2516-2522.

55. Mandi $Y$, Vecsei $L$. The kynurenine system and immunoregulation. J Neural Transm (Vienna) 2012; 119(2): 197-209.

56. Knuesel I, Chicha L, Britschgi M, Schobel SA, Bodmer M, Hellings JA, Toovey S, Prinssen EP. Maternal immune activation and abnormal brain development across CNS disorders. Nat Rev Neurol 2014; 10(11): 643-660.

57. Chiappelli J, Notarangelo FM, Pocivavsek A, Thomas $M A R$, Rowland LM, Schwarcz R, Hong LE. Influence of plasma cytokines on kynurenine and kynurenic acid in schizophrenia. Neuropsychopharmacol 2018; 43(8): 1675-1680.

58. Reus GZ, Becker IRT, Scaini G, Petronilho F, Oses JP, Kaddurah-Daouk R, Ceretta LB, Zugno Al, Dal-Pizzol F, Quevedo J, et al. The inhibition of the kynurenine pathway prevents behavioral disturbances and oxidative stress in the brain of adult rats subjected to an animal model of schizophrenia. Prog Neuropsychopharmacol Biol Psychiatry 2018; 81: 55-63.

59. Moller M, Du Preez JL, Emsley R, Harvey BH. Social isolation rearing in rats alters plasma tryptophan metabolism and is reversed by sub-chronic clozapine treatment. Neuropharmacol 2012; 62(8): 2499-2506.

60. Anderson G, Maes M. Schizophrenia: linking prenatal infection to cytokines, the tryptophan catabolite (TRYCAT) pathway, NMDA receptor hypofunction, neurodevelopment and neuroprogression. Prog Neuropsychopharmacol Biol Psychiatry 2013; 42: 5-19. 